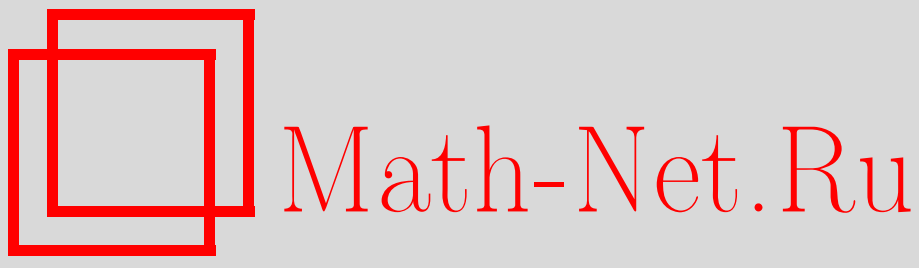

А. А. Архипова, Приграничные априорные оценки для решений недиагональных эллиптических систем с сильной нелинейностью, Изв. РАН. Сер. матем., 2004, том 68 , выпуск 2, 23-38

DOI: https://doi.org/10.4213/im473

Использование Общероссийского математического портала Math-Net.Ru подразумевает, что вы прочитали и согласны с пользовательским соглашением

http://www . mathnet.ru/rus/agreement

Параметры загрузки:

IP: 44.207 .124 .84

26 апреля 2023 г., 17:46:44 
УДК 517.953

А. А. Архипова

\section{Приграничные априорные оценки для решений недиагональных эллиптических систем с сильной нелинейностью}

Рассмотрены квазилинейные эллиптические недиагональные системы уравнений с сильной нелинейностью по градиенту. Ранее автором было установлено, что обобщенное решение такой системы будет непрерывным по Гёльдеру в окрестности тех точек области, где достаточно мала норма градиента решения в пространстве Морри $L^{2, n-2}$. В окрестности таких точек оценена норма Гёльдера решения через его норму в пространстве Соболева $W_{2}^{1}$. Аналогичный результат получен при краевом условии Дирихле для точек, расположенных в окрестности границы области.

Библиография: 10 наименований.

\section{§ 1. Постановка задачи. Формулировка основного результата}

Пусть $\Omega$ - ограниченная область в $\mathbb{R}^{n}, n \geqslant 2$, с достаточно гладкой границей $\partial \Omega$. Пусть $u: \Omega \rightarrow \mathbb{R}^{N}, N>1, u=\left(u^{1}, \ldots, u^{N}\right)$ - решение задачи Дирихле

$$
\begin{gathered}
-\left(A_{k l}^{\alpha \beta}(x, u) u_{x_{\beta}}^{l}\right)_{x_{\alpha}}+b^{k}\left(x, u, u_{x}\right)=0, \quad k=1, \ldots, N, \quad x \in \Omega, \\
\left.u\right|_{\partial \Omega}=0 .
\end{gathered}
$$

Предположим, что матрица $A=\left\{A_{k l}^{\alpha \beta}\right\}_{k, l \leqslant N}^{\alpha, \beta \leqslant n}$ определена на множестве $\mathfrak{M}=$ $\bar{\Omega} \times \mathbb{R}^{N}, b=\left\{b^{k}\right\}^{k \leqslant N}$ - функция Каратеодори на $\mathfrak{M} \times \mathbb{R}^{n N}$ и выполнено следуюшее:

а) условие равномерной эллиптичности

$$
\begin{gathered}
(A(x, u) \xi, \xi)=\sum_{\substack{\alpha, \beta \leqslant n \\
k, l \leqslant N}} A_{k l}^{\alpha \beta}(x, u) \xi_{\alpha}^{k} \xi_{\beta}^{l} \geqslant \nu|\xi|^{2}, \quad \xi \in \mathbb{R}^{n N}, \quad(x, u) \in \mathfrak{M}, \\
\sup _{\mathfrak{M}}\|A(x, u)\| \leqslant \mu ;
\end{gathered}
$$

б) все функции $A_{k l}^{\alpha \beta}$ равномерно непрерывны на $\mathfrak{M}$, более точно, мы предполагаем, что существует непрерывная, ограниченная и неубывающая функция $\omega(s, t)$ такая, что

$$
\|A(x, u)-A(y, v)\| \leqslant \omega\left(|x-y|^{2},|u-v|^{2}\right), \quad x, y \in \bar{\Omega}, \quad u, v \in \mathbb{R}^{N}
$$

Работа выполнена при финансовой поддержке Российского фонда фундаментальных исследований (грант № 02-01-00276). 
при этом $\omega(s, t) \rightarrow 0$, если $s, t \rightarrow 0, \omega$ выпукла по аргументу $t$ при любом фиксированном $s$;

в) условие на порядок роста функции $b$ в форме

$$
|b(x, u, z)| \leqslant a|z|^{2}+\mu, \quad(x, u) \in \mathfrak{M}, \quad z \in \mathbb{R}^{n N}
$$

г) $\partial \Omega \in C^{1}$.

В случае неоднородного условия Дирихле $\left.u\right|_{\partial \Omega}=\varphi$ предполагаем, что функция $\varphi$ достаточно гладкая, и при сведении краевого условия к однородному для преобразованной задачи выполняются условия вида а)-в). Условие (5) определяет сильно нелинейные члены $b\left(x, u, u_{x}\right)$ системы $(1)$. Проблема разрешимости задачи (1) при условии (5) для недиагональных систем остается открытой до настоящего времени. Известны лишь результаты о разрешимости двумерной задачи [1], [2]. В то же время доказана частичная регулярность ограниченных обобщенных решений задачи (1) при условии

$$
2 a\|u\|_{\infty, \Omega}<\nu
$$

(см. работы [3]-[5]).

В локальной постановке проблемы работы [3]-[5] гарантируют, что если $u \in$ $W_{2}^{1}(\Omega) \cap L_{\infty}(\Omega)$ - решение системы $(1)$ и в некотором шаре $B_{R}\left(x^{0}\right) \subset \Omega$

$$
a \underset{B_{R}\left(x^{0}\right)}{\operatorname{Osc}} u<\nu
$$

то $u$ - непрерывная по Гёльдеру функция в $B_{R / 2}\left(x^{0}\right) \backslash \sigma$ и мера Хаусдорфа $H_{n-2-\varepsilon}(\sigma)$ равна нулю при некотором $\varepsilon>0$. Из работы [5] аналогичный результат следует для приграничных шаров $\left(B_{R}\left(x^{0}\right) \cap \Omega \neq B_{R}\left(x^{0}\right)\right)$ при краевых условиях Дирихле и Неймана.

Напомним, что при исследовании регулярности ограниченных обобщенных решений в рамках прямого метода условие (7) использовалось только для того, чтобы доказать повьшение степени интегрируемости решения $u$, точнее, чтобы установить оценку вида

$$
\left\|u_{x}\right\|_{p, B_{R_{1}}\left(x^{0}\right)} \leqslant c\left(1+\left\|u_{x}\right\|_{2, B_{R}\left(x^{0}\right)}\right)
$$

с некоторым $p>2$ при $R_{1}<R ; c=c\left(\nu, \mu, a,\left(R-R_{1}\right)^{-1}\right)$.

Для доказательства непрерывности по Гёльдеру решения в окрестности точки $x^{0}$ дополнительно к (7) предполагалось, что для некоторых чисел $\varepsilon_{0}>0$ и $R_{0}<R_{1}$ справедлива оценка

$$
\oint_{B_{R_{0}}\left(x^{0}\right)}\left|u_{x}\right|^{2} d x \equiv \frac{1}{R_{0}^{n-2}} \int_{B_{R_{0}}\left(x^{0}\right)}\left|u_{x}\right|^{2} d x<\varepsilon_{0},
$$

где число $\varepsilon_{0}$ определяется только данными задачи.

Таким образом, достаточными условиями, гарантирующими непрерывность по Гёльдеру решения в окрестности точки $x^{0}$, являлись предположения (7) и (9). Заметим, что из интегрального тождества для системы (1) следует оценка

$$
\oiint_{B_{R}\left(x^{0}\right)}\left|u_{x}\right|^{2} d x \leqslant \frac{c(\nu, \mu) M_{R}^{2}}{\nu-a M_{R}}, \quad M_{R}=\underset{B_{2 R}\left(x^{0}\right)}{\mathrm{osc}} u .
$$


Таким образом, если полагать, что для $M_{R}$ справедлива оценка

$$
\frac{c(\nu, \mu) M_{R}^{2}}{\nu-a M_{R}}<\varepsilon_{0},
$$

то в окрестности точки $x_{0}$ может быть оценена норма Гёльдера функции $u$.

K сожалению, отсутствие принщипа максимума для недиагональных эллиптических систем уравнений не позволяет получить априорную оценку $L_{\infty}$ нормы решения и, следовательно, проследить случаи, когда условия $(6),(7)$ или $\left(9^{\prime}\right)$ имеют место. Кроме того, согласно указанному описанию точек гёльдеровости решения, точки, для которых не выполнено $(7),(9)$ или $(7),\left(9^{\prime}\right)$, следует отнести к сингулярным точкам решения. Представляется сомнительной возможность оценить в терминах хаусдорфовой размерности все точки $x^{0}$, не удовлетворяющие условию $(7)$. Поэтому мы вынуждены искать другое, более точное описание точек гладкости решения $u$ задачи (1).

В работе автора [6] показано, что условие (7) можно заменить на условие ма-

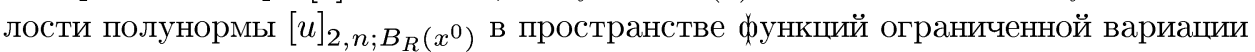
(ВМО). Точнее, в [6] показано, что существует число $\Theta=\Theta(\nu, \mu)>0$ такое, что если

$$
[u]_{2, n ; B_{R}\left(x^{0}\right)}<\Theta \min \left\{\frac{\nu}{a}, 1\right\}
$$

то для некоторых $p=p(\nu, \mu)>2$ и $R_{1}=R_{1}(R)<R$ справедлива оценка (8) с постоянной $c$, зависяшей дополнительно от $\Theta$.

Кроме того, в [6] доказано, что если вместо (10) предположить более сильное условие

$$
\left\|u_{x}\right\|_{L^{2, n-2}\left(B_{R}\left(x^{0}\right)\right)}<\Theta_{1}
$$

для некоторого $\Theta_{1}>0$, то верна не только оценка (8), но и априорная оценка

$$
\|u\|_{C^{\alpha}\left(\overline{B_{R_{2}}\left(x^{0}\right)}\right)} \leqslant c\left(1+\left\|u_{x}\right\|_{2, B_{R_{0}}\left(x^{0}\right)}\right) \quad \forall \alpha \in(0,1), \quad R_{2}<R_{0} .
$$

(Определение нормы в пространстве Морри $L^{2, n-2}\left(B_{R}\right)$ и полунормы $[\cdot]_{2, n ; B_{R}}$ в пространстве $\mathscr{L}^{2, n}\left(B_{R}\right)$ см. ниже в этом параграфе.)

В работе [6] все рассуждения проведены для шаров $B_{R}\left(x^{0}\right)$, лежащих строго внутри области $\Omega$. В то же время, чтобы использовать априорные оценки для доказательства сушествования решения, необходимо иметь оценки не только внутренние, но и приграничные. Для сильно нелинейных систем получение таких оценок связано с определенными техническими трудностями.

Настоящая работа посвящена получению оценки вида (12) в окрестности точки $x^{0} \in \partial \Omega$.

Обобщенным решением задачи (1) назьваем функцию $u \in \stackrel{\circ}{W_{2}^{1}}(\Omega)$, удовлетворяющую тождеству

$$
\int_{\Omega}\left[A_{k l}^{\alpha \beta}(x, u) u_{x_{\beta}}^{l} h_{x_{\alpha}}^{k}+b^{k}\left(x, u, u_{x}\right) h^{k}\right] d x=0 \quad \forall h \in \stackrel{\circ}{C}^{1}(\bar{\Omega}) .
$$

ЗАмЕчАниЕ 1 . Получаемые в настояшей работе априорные оценки предполагается использовать для доказательства существования обобшенного решения задачи (1), при этом мы будем рассматривать некоторые регуляризации задачи, и предположение о дополнительной гладкости решения в окрестности исследуемой точки не является для нас обременительным.

Основным результатом работы является следуюшая теорема. 
ТЕОРема 1. Пусть выполнены условия а)-г) $u и \in \stackrel{\circ}{\mathrm{W}} \frac{1}{2}(\Omega)$ - обобщенное решение задачи (1). Существует такое число $\Theta=\Theta(\nu, \mu, n)>0$, что если для некоторой точки $x^{0} \in \bar{\Omega}$ найдется $R_{0}>0$ такое, ито $u \in C^{1}\left(\overline{\Omega_{R_{0}}\left(x^{0}\right)}\right) u$

$$
\left\|u_{x}\right\|_{L^{2, n-2}\left(\Omega_{R_{0}}\left(x^{0}\right)\right)}<\Theta \min \left\{\frac{\nu}{a}, 1\right\},
$$

то имеет место оценка

$$
\|u\|_{C^{\alpha}\left(\overline{\Omega_{R_{\star}}\left(x^{0}\right)}\right)} \leqslant c\left(1+\|u\|_{W_{2}^{1}\left(\Omega_{R_{0}}\left(x^{0}\right)\right)}\right) \quad \forall \alpha \in(0,1)
$$

при некотором $R_{\star}<R_{0}$.

Постоянные с и $R_{\star}$ в оценке (15) определяются параметрами $\nu, \mu, a, \alpha$, модулем непрерьвности $\omega(\cdot, \cdot)$ из условия б), а также $C^{1}$-характеристикой $\Omega \Omega$.

ЗАМЕЧАНИЕ 2. Предположение теоремы 1 о принадлежности $u(x)$ пространству $C^{1}\left(\overline{\Omega_{R_{0}}\left(x^{0}\right)}\right)$ можно ослабить и считать, что $u_{x} \in L^{m}\left(\Omega_{R_{0}}\right) \cap L^{2, n-2}\left(\Omega_{R_{0}}\right)$ с каким либо $m>2$ (см. замечание 1$)$.

ЗАмЕчАНИЕ 3. Чтобы доказать теорему, достаточно проанализировать случай $x^{0} \in \partial \Omega$. Далее, учитывая внутренние оценки, установленные в [6], и "сшивая" приграничные оценки с внутренними, получим справедливость теоремы 1 в обшем случае.

В настоящей работе приняты следующие обозначения: $x=\left(x_{1}, \ldots, x_{n}\right) \in \mathbb{R}^{n}$, $n \geqslant 2$;

$$
\begin{aligned}
& Q_{r}\left(x^{0}\right)=\left\{x \in \mathbb{R}^{n}:\left|x_{i}-x_{i}^{0}\right|<r, i=1, \ldots, n\right\}, \\
& B_{r}\left(x^{0}\right)=\left\{x \in \mathbb{R}^{n}:\left|x-x^{0}\right|<r\right\}, \quad S_{r}\left(x^{0}\right)=\partial B_{r}\left(x^{0}\right) ;
\end{aligned}
$$

$\Omega$ - ограниченная область в $\mathbb{R}^{n}, \Omega_{\rho}\left(x^{0}\right)=\Omega \cap B_{\rho}\left(x^{0}\right) ;|A|=\operatorname{meas}_{n} A-$ мера Лебега множества $A \subset \mathbb{R}^{n}$,

$$
\begin{gathered}
\oint_{A} f(x) d x=\frac{1}{|A|} \int_{A} f(x) d x, \quad U_{\rho, x^{0}}=\oint_{\Omega_{\rho}\left(x^{0}\right)} U(x) d x \\
\|V\|_{m, A}=\|V\|_{L^{m}(A)}, \quad m \in[1, \infty]
\end{gathered}
$$

Напомним, что в пространствах Морри $L^{2, \alpha}(D), \alpha \in(0, n]$, где $D$ - ограниченное множество в $\mathbb{R}^{n}$, норма определяется следующим образом:

$$
\begin{gathered}
\|U\|_{L^{2, \alpha}(D)}=\left(\sup _{\substack{x^{0} \in D \\
\rho \leqslant d}} \frac{1}{\rho^{\alpha}} \int_{D_{\rho}\left(x^{0}\right)}|U|^{2} d x\right)^{\frac{1}{2}}, \\
D_{\rho}\left(x^{0}\right)=D \cap B_{\rho}\left(x^{0}\right), \quad d=\operatorname{diam} D .
\end{gathered}
$$

В пространствах Кампанато $\mathscr{L}^{2, \beta}(D), \beta \in(0, n+2]$, норма определяется следующим образом:

$$
\|U\|_{\mathscr{L}^{2, \beta}(D)}^{2}=\|U\|_{2, D}^{2}+[U]_{2, \beta ; D}^{2}
$$


где

$$
[U]_{2, \beta ; D}=\left(\sup _{\substack{x^{0} \in D \\ \rho \leqslant d}} \frac{1}{\rho^{\beta}} \int_{D_{\rho}\left(x^{0}\right)}\left|U-U_{\rho, x^{0}}\right|^{2} d x\right)^{\frac{1}{2}}
$$

- полунорма.

Если $D$ - область muna (A), т. е. сушествует число $A>0$ такое, что

$$
\left|D_{\rho}(x)\right| \geqslant A \rho^{n}, \quad x \in D, \quad \forall \rho \leqslant d,
$$

то пространства $\mathscr{L}^{2, \beta}(D)$ при $\beta=n+2 \gamma, \gamma \in(0,1)$, изоморфны пространствам Гёльдера $C^{\gamma}(\bar{D})$.

Отметим также, что пространства $\mathscr{L}^{m, n}(Q)$, где $Q-$ куб в $\mathbb{R}^{n}$, при различных $m \geqslant 1$ изоморфны между собой и определяют пространство $\mathrm{BMO}(Q)$, кроме того, эти пространства совпадают с пространством Йона-Ниренберга $\mathrm{JN}(Q)$. (О свойствах пространств $L^{p, \beta}, \mathscr{L}^{p, \beta}$ и JN см. [7, гл. IV], [8, гл. I] и [9]). При доказательстве теоремы 1 будет существенно использоваться теорема о квазиобратных неравенствах Гёльдера, доказанная автором в [6]. Сформулируем ее.

ТеОРема 2 [6, теорема 1]. Пусть фиксированьи числа $q, l>1, \omega>0$ и число $m>q l$. Пусть $g \in L^{m}\left(Q_{r}(\widehat{x})\right)$ удовлетворяет неравенствам

$$
\oint_{B_{\frac{r}{2}}} g^{q} d x \leqslant c_{0}\left[\left(\oint_{B_{r}} g d x\right)^{q}+b \omega\left(\oint_{B_{r}} g^{q l} d x\right)^{\frac{1}{l}}\right] \quad \forall B_{r} \subset Q_{r}(\widehat{x})
$$

где $c_{0}, b$ - положстельные постоянные и $b$ может зависеть от $l$.

Существует число $p_{0}>q$, зависящее только от $q$ и $c_{0}$, такое, что для $p \in\left(q, \min \left\{p_{0}, m\right\}\right)$

$$
\left(f_{Q_{\frac{r}{2}}} g^{p} d x\right)^{\frac{1}{p}} \leqslant c_{\star}\left(\oint_{Q_{r}} g^{q} d x\right)^{\frac{1}{q}} \quad \forall Q_{r} \subset Q_{R}(\widehat{x})
$$

если в неравенствах $(16) l \in(1, p / q), \quad \omega<\kappa_{0} / b, \kappa_{0}$ - некоторое число, зависящее от $p, q, l u c_{0}$.

В частности,

$$
\|g\|_{p, Q_{r}(\widehat{x})} \leqslant c_{\star \star}\|g\|_{q, Q_{R}(\widehat{x})}, \quad r<R .
$$

Постоянные $c_{\star} u c_{\star \star}$ зависят от $p, q, l, c_{0}, b(l) u \omega$, a $c_{\star \star}$ зависит также om $(R-r)^{-1}$.

ЗАмЕчАнИЕ 4. Из анализа доказательства теоремы 1 , проведенного в [6] (в частности, анализа доказательства леммы 1 из [6]), следует, что постоянные $c_{\star}$ и $c_{\star \star}$ в теореме 2 можно считать зависящими от $\kappa_{0}$, а не от $\omega$. 


\section{§2. Доказательство теоремы 1}

Докажем сначала две леммы. В первой лемме при условии (14) устанавливается $L^{p}$-оценка градиента решения при некотором $p>2$. Во второй лемме мы получаем оценку $C^{\alpha}$-нормы решения в окрестности точки $x_{0} \in \partial \Omega$ через $W_{2}^{1}$-норму решения.

Лемма 1. Пусть выполнены условия теоремы 1 , причем $x^{0} \in \partial \Omega$. Тогда существует такое число $s=s(\nu, \mu)>2$, что

$$
\left\|u_{x}\right\|_{s, \Omega_{\widetilde{R}}\left(x^{0}\right)} \leqslant c_{1}\left(1+\left\|u_{x}\right\|_{2, \Omega_{R_{0}}\left(x^{0}\right)}\right)
$$

при некотором $\widetilde{R}<R_{0}$, число $R_{0}-$ из условия (14).

В частности, справедливы неравенства

$$
\left(\oint_{\Omega_{\rho}(y)}\left(1+\left|u_{x}\right|\right)^{s} d x\right)^{\frac{2}{s}} \leqslant c_{2} \oint_{\Omega_{t \rho}(y)}\left(1+\left|u_{x}\right|\right)^{2} d x \quad \forall \Omega_{t \rho}(y) \subset \Omega_{R_{0}}\left(x^{0}\right),
$$

где $t=t(n)>2$. Постоянные $c_{1}$ и $c_{2}$ зависят от $\nu, \mu$, a и $n$, а $c_{1}$, кроме того, зависит от $\left(R_{0}-\widetilde{R}\right)^{-1}$.

ДоКАЗАТЕЛЬСТВО. Зафиксируем произвольно $x^{0} \in \partial \Omega$, положим $R_{1}=$ $R_{0} / \sqrt{n}$, где $R_{0}$ - из условия (14). Пусть сначала $y^{0} \in \partial \Omega \cap B_{R_{1}}\left(x^{0}\right), B_{r}\left(y^{0}\right) \subset$ $B_{R_{1}}\left(x^{0}\right)$. Рассмотрим тождество $(13)$ с $h=u \xi^{2}$, где $\xi$ - срезающая для $B_{r}\left(y^{0}\right)$ функция, $\left.h\right|_{\partial \Omega_{r}\left(y^{0}\right)}=0$. Получаем неравенство

$$
\int_{\Omega_{\frac{r}{2}\left(y^{0}\right)}}\left|u_{x}\right|^{2} d x \leqslant \frac{4 \mu}{\nu r^{2}} \int_{\Omega_{r}\left(y^{0}\right)}|u|^{2} d x+\frac{2 a}{\nu} \int_{\Omega_{r}\left(y^{0}\right)}\left|u_{x}\right|^{2}|u| d x+\frac{2 \mu}{\nu}\left|B_{r}\right| .
$$

Заметим, что $\left.u\right|_{\gamma_{r}\left(y^{0}\right)}=0$, где $\gamma_{r}\left(y^{0}\right)=B_{r}\left(y^{0}\right) \cap \partial \Omega$. В силу теоремы вложения пространств $W_{2 n /(n+2)}^{1}\left(\Omega_{r}\right) \hookrightarrow L^{2}\left(\Omega_{r}\right)$ верна оценка

$$
\int_{\Omega_{r}}|u|^{2} d x \leqslant c_{3}\left(\int_{\Omega_{r}}\left|u_{x}\right|^{\frac{2 n}{n+2}} d x\right)^{\frac{n+2}{n}}
$$

Нам предстоит оценить в (21) интеграл $J_{r}=\int_{\Omega_{r}\left(y^{0}\right)}\left|u_{x}\right|^{2}|u| d x$. Пусть $\widetilde{u}-$ продолженная нулем вне $\Omega$ функция $u$; тогда $\widetilde{u} \in W_{2}^{1}\left(B_{r}\left(y^{0}\right)\right)$ и для любого $s>1$

$$
\begin{gathered}
\left(\int_{\Omega_{r}\left(y^{0}\right)}\left|u-u_{r, y^{0}}\right|^{s} d x\right)^{\frac{1}{s}} \leqslant\left(1+\left(\frac{\left|B_{r}\right|}{\left|\Omega_{r}\right|}\right)^{1-\frac{1}{s}}\right)\left(\int_{B_{r}\left(y^{0}\right)}\left|\widetilde{u}-\widetilde{u}_{r, y^{0}}\right|^{s} d x\right)^{\frac{1}{s}}, \\
\widetilde{u}_{r, y^{0}}=\oint_{B_{r}\left(y^{0}\right)} \widetilde{u} d x .
\end{gathered}
$$

Поскольку $\partial \Omega \in C^{1}$, то величина $1+\left(\left|B_{r}\right| /\left|\Omega_{r}\right|\right)^{1-1 / s}$ оценивается в неравенстве (23) постоянной $c_{4}$. 
Для произвольно фиксированного $l>1$ и $l^{\prime}=l /(l-1)$ справедливы неравенства

$$
\begin{aligned}
J_{r} \leqslant & \left(\oint_{\Omega_{r}\left(y^{0}\right)}\left|u_{x}\right|^{2 l} d x\right)^{\frac{1}{l}}\left(\oint_{\Omega_{r}\left(y^{0}\right)}|u|^{l^{\prime}} d x\right)^{\frac{1}{l^{\prime}}}\left|\Omega_{r}\right| \\
\leqslant & \left|\Omega_{r}\right|\left(\oint_{\Omega_{r}\left(y^{0}\right)}\left|u_{x}\right|^{2 l} d x\right)^{\frac{1}{l}}\left\{\left|u_{r, y^{0}}\right|+\left(\oint_{\Omega_{r}\left(y^{0}\right)}\left|u-u_{r, y^{0}}\right|^{l^{\prime}} d x\right)^{\frac{1}{l^{\prime}}}\right\} \\
\leqslant & \left|\Omega_{r}\right|\left(\oint_{\Omega_{r}\left(y^{0}\right)}\left|u_{x}\right|^{2 l} d x\right)^{\frac{1}{l}} \\
& \times c_{4}\left\{\left(\oint_{\Omega_{r}\left(y^{0}\right)}\left|u_{x}\right|^{2} d x\right)^{\frac{1}{2}}+\left(\oint_{B_{r}\left(y^{0}\right)}\left|\widetilde{u}-\widetilde{u}_{r, y^{0}}\right|^{l^{\prime}} d x\right)^{\frac{1}{l^{\prime}}}\right\} \\
& \stackrel{(14)}{\leqslant}\left|\Omega_{r}\right|\left(\oint_{\Omega_{r}\left(y^{0}\right)}\left|u_{x}\right|^{2 l} d x\right)^{\frac{1}{l}}\left\{\Theta \min \left\{\frac{\nu}{a}, 1\right\} c_{4}+c_{4}[\widetilde{u}]_{l^{\prime}, n ; Q_{R_{1}}\left(x^{0}\right)}\right\} \\
\leqslant & \left|\Omega_{r}\right|\left(\oint_{\Omega_{r}}\left|u_{x}\right|^{2 l} d x\right)^{\frac{1}{l}}\left\{\Theta \min \left\{\frac{\nu}{a}, 1\right\} c_{4}+c_{5}(l)[\widetilde{u}]_{2, n ; Q_{R_{1}}\left(x^{0}\right)}\right\} .
\end{aligned}
$$

В этой цепочке неравенств условие (14) использовано пока с произвольным $\Theta$. Здесь и далее мы обозначаем

$$
\oint_{\Omega_{r}}\left|u_{x}\right|^{2} d x=\frac{1}{r^{n-2}} \int_{\Omega_{r}}\left|u_{x}\right|^{2} d x .
$$

Последнее неравенство в соотношениях (24) справедливо в силу изоморфизма пространств Кампанато $\mathscr{L}^{m, n}\left(Q_{R_{1}}\right)$ при различных $m \geqslant 1$ и оценки

$$
[v]_{m, n ; Q_{R_{1}}} \leqslant c(m, n)[v]_{2, n ; Q_{R_{1}}} \quad \forall v \in \mathscr{L}^{2, n}\left(Q_{R_{1}}\right) .
$$

Поскольку

$$
[\widetilde{u}]_{2, n ; Q_{R_{1}}} \leqslant c\left\|\widetilde{u}_{x}\right\|_{L^{2, n-2}\left(Q_{R_{1}}\right)} \leqslant c\left\|u_{x}\right\|_{L^{2, n-2}\left(\Omega_{R_{0}}\right)} \stackrel{(14)}{\leqslant} c_{6} \Theta \min \left\{\frac{\nu}{a}, 1\right\},
$$

то для $J_{r}$ получаем оценку

$$
J_{r} \leqslant c_{7}(l)\left|\Omega_{r}\right| \Theta \min \left\{\frac{\nu}{a}, 1\right\}\left(\oint_{\Omega_{r}\left(y^{0}\right)}\left|u_{x}\right|^{2 l} d x\right)^{\frac{1}{l}} .
$$

Теперь из неравенства (21) с помошью оценок (22) и (25) выводим для $g(x)=$ $\left(1+\left|u_{x}\right|\right)^{2 n /(n+2)}$ оценку $(16)$ с заменой $B_{r}$ на $\Omega_{r}$, при этом $q=(n+2) / n, \omega=\Theta$, $R=R_{1}, \widehat{x}=x^{0}$.

Пусть $y^{0} \in \Omega_{r}\left(x^{0}\right), d=\operatorname{dist}\left(y^{0}, \partial \Omega\right)>0$. Зафиксируем произвольно $B_{r}\left(y^{0}\right) \subset$ $\Omega_{R_{1}}\left(x^{0}\right), r \leqslant d$, и рассмотрим тождество (13) с $h=\left(u-u_{r, y^{0}}\right) \xi^{2}, \xi$ - срезаюшая для $B_{r}\left(y^{0}\right)$ функция, $\xi=1$ в $B_{r / 2}\left(y^{0}\right)$. Из полученного соотношения выводим неравенство

$$
\begin{aligned}
\int_{B_{\frac{r}{2}}\left(y^{0}\right)}\left|u_{x}\right|^{2} d x \leqslant & \frac{4 \mu}{\nu r^{2}} \int_{B_{r}\left(y^{0}\right)}\left|u-u_{r, y^{0}}\right|^{2} d x \\
& +\frac{2 a}{\nu} \int_{B_{r}\left(y^{0}\right)}\left|u_{x}\right|^{2}\left|u-u_{r, y^{0}}\right| d x+\frac{2 \mu}{\nu}\left|B_{r}\right| .
\end{aligned}
$$


Как и в предыдушем случае, из этого неравенства следует, что

$$
\begin{gathered}
\int_{B_{\frac{r}{2}}\left(y^{0}\right)}\left|u_{x}\right|^{2} d x \leqslant \frac{c_{8}}{r^{2}}\left(\int_{B_{r}\left(y^{0}\right)}\left|u_{x}\right|^{\frac{2 n}{n+2}} d x\right)^{\frac{n+2}{n}}+\frac{2 a}{\nu} J_{r}+\frac{2 \mu}{\nu}\left|B_{r}\right|, \\
J_{r}=\int_{B_{r}\left(y^{0}\right)}\left|u_{x}\right|^{2}\left|u-u_{r, y^{0}}\right| d x .
\end{gathered}
$$

Рассуждая так же, как при выводе оценки (25), получаем цепочку неравенств

$$
\begin{aligned}
J_{r} & \leqslant\left|B_{r}\right|\left(\oint_{B_{r}}\left|u_{x}\right|^{2 l} d x\right)^{\frac{1}{l}}\left(\oint_{B_{r}}\left|u-u_{r, y^{0}}\right|^{l^{\prime}} d x\right)^{\frac{1^{\prime}}{l^{\prime}}} \\
& \leqslant c\left|B_{r}\right|[u]_{l^{\prime}, n ; Q_{R_{1}}\left(x^{0}\right)}\left(\oint_{B_{r}}\left|u_{x}\right|^{2 l} d x\right)^{\frac{1}{l}} \\
& \leqslant c(l)\left|B_{r}\right|[u]_{2, n ; Q_{R_{1}}\left(x^{0}\right)}\left(\oint_{B_{r}}\left|u_{x}\right|^{2 l} d x\right)^{\frac{1}{l}} \\
& \leqslant c(l)\left|B_{r}\right|\left\|u_{x}\right\|_{L^{2, n-2}\left(B_{R_{0}}\left(x^{0}\right)\right)}\left(\oint_{B_{r}}\left|u_{x}\right|^{2 l} d x\right)^{\frac{1}{l}} \\
& \stackrel{(14)}{\leqslant} c(l)\left|B_{r}\right| \Theta \min \left\{\frac{\nu}{a}, 1\right\}\left(\oint_{B_{r}}\left|u_{x}\right|^{2 l} d x\right)^{\frac{1}{l}} .
\end{aligned}
$$

Из $(26)$ и оценки для $J_{r}$ следует неравенство

$$
\begin{gathered}
\oint_{B_{\frac{r}{2}}} g^{q} d x \leqslant c_{9}\left[\left(\oint_{B_{r}} g d x\right)^{q}+c_{10}(l) \Theta\left(f_{B_{r}} g^{q l} d x\right)^{\frac{1}{l}}\right] \\
g=\left(1+\left|u_{x}\right|\right)^{\frac{2 n}{n+2}}, \quad q=\frac{n+2}{n} .
\end{gathered}
$$

"Сшивая" теперь внутреннюю и приграничную оценки для функции $g$, получим неравенства вида $(27)$ в $\Omega_{r}\left(y^{0}\right), \Omega_{t r}\left(y^{0}\right)$ при любом расположении точек $y^{0}$ в $\overline{\Omega_{R_{1}}\left(x^{0}\right)}$ и при любом $r$ таком, что $B_{t r}\left(y^{0}\right) \subset B_{R_{1}}\left(x^{0}\right), t \geqslant 2$-абсолютная постоянная.

Кроме того, полагая

$$
\widetilde{g}(x)= \begin{cases}g(x), & x \in \Omega_{R_{0}}\left(x^{0}\right), \\ 0, & x \in B_{R_{0}}\left(x^{0}\right) \backslash \Omega_{R_{0}}\left(x^{0}\right),\end{cases}
$$

нетрудно вывести неравенства

$$
\oint_{B_{\rho}} \widetilde{g}^{q} d x \leqslant c_{11}(\nu, \mu)\left[\left(\oint_{B_{t \rho}} \tilde{g} d x\right)^{q}+c_{12}(l) \Theta\left(\oint_{B_{t \rho}} \widetilde{g}^{q l} d x\right)^{\frac{1}{l}}\right] \quad \forall B_{t \rho} \subset B_{R_{1}}\left(x^{0}\right),
$$

где $t \geqslant 2$ - некоторая абсолютная постоянная.

Неравенства (28) названы автором квазиобратными неравенствами Гёльдера (при $c_{12}=0$ это хорошо известные обратные неравенства Гёльдера). 
Согласно теореме 2 сушествует число $p_{0}=p_{0}\left(\mathrm{c}_{11}, q\right)>q$ такое, что для любого $p \in\left(q, p_{0}\right)$ верна оценка

$$
\|\widetilde{g}\|_{p, Q_{R}\left(x^{0}\right)} \leqslant c_{13}\|\widetilde{g}\|_{q, Q_{\widehat{R}}\left(x^{0}\right)}, \quad R<\widehat{R}=\frac{R_{1}}{\sqrt{n}},
$$

если в неравенствах (28) фиксировать $l \in(1, p / q)$ и считать, что $c_{12}(l) \Theta<\kappa_{0}$, где $\kappa_{0}$ - некоторое число, зависящее от $p, q, l, a$ и $c_{11}$. Если, например, зафиксировать $l=(q+p) /(2 q)$, то нетрудно видеть, что $\kappa_{0}$ определяется только параметрами $\nu, \mu, a$ и $n$. Параметр $\Theta$ в условии (14) можно фиксировать из условия $\Theta=\kappa_{0} /\left(2 c_{12}\right)$. Число $\Theta$ зависит от тех же величин, что и $\kappa_{0}, \widehat{R}=R_{0} / n$. Вместе с оценкой (29) выполняются неравенства

$$
\left(\oint_{Q_{\frac{r}{2}}} \widetilde{g}^{p} d x\right)^{\frac{1}{p}} \leqslant c_{14}\left(\oint_{Q_{r}} \widetilde{g}^{q} d x\right)^{\frac{1}{q}} \quad \forall Q_{r} \subset Q_{\widehat{R}}\left(x^{0}\right) .
$$

Постоянные $c_{13}$ и $c_{14}$ зависят от $q, c_{11}, p, l=(q+p) /(2 q), \kappa_{0}$ и, следовательно, от $\nu, \mu, a$ и $n ; c_{13}$ зависит, кроме того, от $(\widehat{R}-R)^{-1}$. Учитывая определения функций $\widetilde{g}, g$ и переходя от интегрирования по кубам $Q_{\rho}$ к интегрированию по шарам $B_{\rho}$, из (29) и (30) выводим неравенства (19) и (20).

ЗамечаниЕ 5. Далее число $\Theta$ из условия (14), при котором имеют место оценки (19) и (20), будем обозначать $\Theta_{1}$.

ЛЕмма 2. Пусть выполнены условия теоремы 1 и $x^{0} \in \partial \Omega$. Существует такое число $\Theta_{2}=\Theta_{2}(\nu, \mu, n)>0$, что если условие (14) выполнено при $\Theta=\Theta_{2}, m o$

$$
\|u\|_{C^{\alpha}\left(\overline{\left.\Omega_{R_{2}}\left(x^{0}\right)\right)}\right.} \leqslant c_{15}\left(1+\|u\|_{W_{2}^{1}\left(\Omega_{R_{0}}\left(x^{0}\right)\right)}\right) \quad \forall \alpha \in(0,1),
$$

радиус $R_{2}<R_{0}$ и постоянная $c_{15}$ зависят от $\nu, \mu, a, \alpha, n$, модуля непрерывности $\omega(\cdot, \cdot)$ из условия б) теоремы 1 и $C^{1}$-характеристики $\partial \Omega$ (число $R_{0}-$ из условия (14)).

ДокАЗАТЕльСТво. Будем пока считать, что условие (14) выполнено с произвольным $\Theta_{2} \leqslant \Theta_{1}$, где $\Theta_{1}$ - параметр, для которого верна лемма 1. Таким образом, в $\Omega_{R_{0}}\left(x^{0}\right)$, где $x^{0} \in \partial \Omega$, справедливы неравенства (20) с некоторым $s>2$.

Прежде всего, мы "распрямим" часть поверхности $\partial \Omega$ в окрестности точки $x^{0}$. Существует окрестность $V$ точки $x^{0}, V \subset B_{R_{0}}\left(x^{0}\right)$, на которой определен диффеоморфизм $y=y(x)$, переводяший множество $V \cap \Omega$ в $B_{2}^{+}(0)$ так, что $y(V \cap \partial \Omega)=\Gamma_{2}$, $\Gamma_{r}=B_{r}(0) \cap\left\{y_{n}=0\right\}$. В новых переменных локально задача (1) примет вид

$$
\begin{gathered}
-\frac{d}{d y_{\gamma}}\left(\mathfrak{O}_{k l}^{\gamma \delta}(y, \widetilde{u}) \widehat{u}_{y_{\delta}}^{l}\right)+\mathbb{B}^{k}\left(y, \widehat{u}, \widehat{u}_{y}\right)=0, \quad y \in B_{2}^{+}, \\
\left.\widehat{u}\right|_{\Gamma_{2}}=0 .
\end{gathered}
$$

Здесь $\widehat{u}(y)=u(x(y)), \mathfrak{O}_{k l}^{\gamma \delta}(y, \widetilde{u})=A_{k l}^{\alpha \beta}(x(y), u(x(y))) \frac{\partial y_{\delta}(x(y))}{\partial x_{\beta}} \cdot \frac{\partial y_{\gamma}(x(y))}{\partial x_{\alpha}} I(y)$, $\mathbb{B}^{k}\left(y, \widehat{u}, \widehat{u}_{y}\right)=b^{k}\left(x(y), \widehat{u}(y), \widehat{u}_{y} \frac{\partial y(x(y))}{\partial x}\right) I(y), I(y)=\left|\operatorname{det}\left\{\frac{\partial x(y)}{\partial y}\right\}\right|>0, y \in \overline{B_{2}^{+}}$. 
Нетрудно видеть, что для задачи (32) вьполнены условия вида а)-в) с другими постоянными $\widehat{\nu}, \widehat{\mu}, \widehat{a}$ и функцией $\widehat{\omega}(\cdot, \cdot)$, которые определяются параметрами $\nu, \mu, a$, функцией $\omega(\cdot, \cdot)$, а также величиной $c_{\Gamma}$. Здесь и далее через $c_{\Gamma}$ обозначены различные постоянные, определяемые $C^{1}$-нормами преобразований $y=y(x)$ и $x=x(y)$ на множествах $\overline{V \cap \Omega}$ и $\overline{B_{2}^{+}}$соответственно. Из неравенства (14) для функции $\widehat{u}$ следует оценка

$$
\left\|\widehat{u}_{y}\right\|_{L^{2, n-2}\left(B_{2}^{+}\right)} \leqslant c_{16} \Theta_{2}, \quad c_{16}=c_{16}\left(c_{\Gamma}\right)
$$

а из неравенств (20) получаем соотношения

$$
\left(\oint_{\omega_{\rho}(y)}\left(1+\left|\widehat{u}_{y}\right|\right)^{s} d y\right)^{\frac{2}{s}} \leqslant c_{17} \oint_{\omega_{\lambda \rho}(y)}\left(1+\left|\widehat{u}_{y}\right|\right)^{2} d y \quad \forall y \in \overline{B_{\frac{3}{2}}^{+}}, \quad \lambda \rho \leqslant \frac{1}{2} .
$$

Здесь $\omega_{r}(y)=B_{r}(y) \cap B_{2}^{+}$, постоянная $c_{17}$ зависит от $\nu, \mu, a, n$ и $c_{\Gamma}$, a $\lambda=$ $\lambda\left(c_{\Gamma}, n\right) \geqslant 2$.

Зафиксируем произвольно точку $y^{0} \in \overline{B_{1}^{+}(0)}$ и радиус $R \leqslant 1 /(2 \lambda)$. Пусть $v-$ решение модельной задачи

$$
\begin{gathered}
\mathfrak{O}_{k l}^{\gamma \delta}\left(y^{0}, \widehat{u}^{0}\right) v_{y_{\delta} y_{\gamma}}^{l}=0 \quad \text { в } \omega_{R}\left(y^{0}\right), \\
\left.v\right|_{\Gamma_{R}\left(y^{0}\right)}=0,\left.\quad v\right|_{S_{R}^{\prime}\left(y^{0}\right)}=\widehat{u},
\end{gathered}
$$

$\Gamma_{R}\left(y^{0}\right)=B_{R}\left(y^{0}\right) \cap \Gamma_{2}, \quad S_{R}^{\prime}\left(y^{0}\right)=\partial \omega_{R}\left(y^{0}\right) \backslash \Gamma_{R}\left(y^{0}\right), \quad \widehat{u}^{0}=\oint_{\omega_{R}\left(y^{0}\right)} \widehat{u} d y$.

Для решения $v$ задачи (35) справедлива оценка Кампанато

$$
\int_{\omega_{\rho}\left(y^{0}\right)}\left|v_{y}\right|^{2} d y \leqslant c_{18}\left(\frac{\rho}{R}\right)^{n} \int_{\omega_{R}\left(y^{0}\right)}\left|v_{y}\right|^{2} d y \quad \forall \rho \leqslant R
$$

с постоянной $c_{18}=c_{18}(\widehat{\nu}, \widehat{\mu})$.

Из интегрального тождества для функции $v$ следует, что

$$
\left\|v_{y}\right\|_{2, \omega_{R}\left(y^{0}\right)} \leqslant c_{19}(\widehat{\nu}, \widehat{\mu})\left\|\widehat{u}_{y}\right\|_{2, \omega_{R}\left(y^{0}\right)} .
$$

Кроме того, нетрудно показать, что градиент функции $v$ удовлетворяет в $\omega_{R}\left(y^{0}\right)$ обратным неравенствам Гёльдера, при этом функция $\widehat{u}$, определяющая условие Дирихле для $v$, принадлежит пространству $W_{s}^{1}\left(B_{3 / 2}^{+}\right), s>2$. Отсюда следует, что при некотором $p>2$ для функции $v$ в $\omega_{R}\left(y^{0}\right)$ справедлива глобальная оценка

$$
\left(\oint_{\omega_{R}\left(y^{0}\right)}\left|v_{y}\right|^{p} d y\right)^{\frac{2}{p}} \leqslant c_{20}\left\{\oint_{\omega_{R}\left(y^{0}\right)}\left|v_{y}\right|^{2} d y+\left(\oint_{\omega_{R}\left(y^{0}\right)}\left|\widehat{u}_{y}\right|^{p} d y\right)^{\frac{2}{p}}\right\} .
$$

Предполагая, что $p \leqslant s(s>2$ - из оценки (34)), из неравенств $(38),(34)$ и $(37)$ выводим оценку

$$
\left(\oint_{\omega_{R}\left(y^{0}\right)}\left|v_{y}\right|^{p} d y\right)^{\frac{2}{p}} \leqslant c_{21} \oint_{\omega_{\lambda R}\left(y^{0}\right)}\left(1+\left|\widehat{u}_{y}\right|^{2}\right) d y
$$

с постоянной $c_{21}$, зависяшей от $\nu, \mu, a, n$ и $c_{\Gamma}$. 
Определим функцию $w=\widehat{u}-v,\left.w\right|_{\partial \omega_{R}\left(y^{0}\right)}=0$. Для нее из интегральных тождеств, соответствуюших задачам (32) и (35), выводим соотношение

$$
\int_{\omega_{R}\left(y^{0}\right)}\left[\mathfrak{O}_{k l}^{\gamma \delta}\left(y^{0}, \widehat{u}^{0}\right) w_{y_{\delta}}^{l} h_{y_{\gamma}}^{k}+\mathbb{B}^{k}\left(y, \widehat{u}, \widehat{u}_{y}\right) h^{k}+\Delta \mathfrak{O}_{k l}^{\gamma \delta} \widehat{u}_{y_{\delta}}^{l} h_{y_{\gamma}}^{k}\right] d y=0
$$

где $\Delta \mathfrak{O}_{k l}^{\gamma \delta}=\mathfrak{O}_{k l}^{\gamma \delta}(y, \widehat{u})-\mathfrak{O}_{k l}^{\gamma \delta}\left(y^{0}, \widehat{u}^{0}\right)$.

Заметим, что в случае, когда $\omega_{R}\left(y^{0}\right) \neq B_{R}\left(y^{0}\right)$, мы не можем из тождества (40) оценить интеграл $\int_{\omega_{R}\left(y^{0}\right)}\left|w_{y}\right|^{2} d y$ так, как это сделано в [6] при $\omega_{R}\left(y^{0}\right)=B_{R}\left(y^{0}\right)$. В данном случае мы имеем область с незладкой гранищей и не можем воспользоваться теорией регулярности решений линейных систем в пространствах Морри и Кампанато (см. вывод оценки (2.27) для задачи (2.24) в [6]). Тем не менее, мы покажем, как можно оценить сверху интеграл

$$
\int_{\omega_{t R}\left(y^{0}\right)}\left|w_{y}\right|^{2} d y, \quad t=\frac{3}{4 \sqrt{n}}
$$

подбором подходящей функции $h$ в тождество (40).

Зафиксируем пока произвольно числа $0<s \ll 1$ и $T \gg 1$, положим в тождестве (40) $h=w\left((2 T)^{s}-|w|^{s}\right)_{+}=w \max \left\{(2 T)^{s}-|w|^{s}, 0\right\}$. Функция $h$ ограничена на $\omega_{R}\left(y^{0}\right),|h| \leqslant(2 T)^{s+1},\left.h\right|_{\partial \omega_{R}\left(y^{0}\right)}=0$. Из полученного соотношения следует неравенство

$$
\begin{aligned}
& \frac{\widetilde{\nu}}{2} \int_{\omega_{R}\left(y^{0}\right)}\left|w_{y}\right|^{2}\left((2 T)^{s}-|w|^{s}\right)_{+} d y \leqslant c_{22}\left[s \int_{\omega_{R}\left(y^{0}\right)}\left|w_{y}\right|^{2}|w|^{s} \chi_{+} d y\right. \\
& \quad+\int_{\omega_{R}\left(y^{0}\right)}\left(1+\left|\widehat{u}_{y}\right|\right)^{2}|w|\left((2 T)^{s}-|w|^{s}\right)_{+} d y \\
& \left.+T^{s} \int_{\omega_{R}\left(y^{0}\right)}|\Delta \mathfrak{O}|^{2}\left|\widehat{u}_{y}\right|^{2} d y\right]
\end{aligned}
$$

где $\chi_{+}-$характеристическая функция множества $\left\{y \in \omega_{R}\left(y^{0}\right):|w(y)| \leqslant 2 T\right\}$.

Зафиксируем числа $\tau=T / 2^{1 / s}, t=3 /(4 \sqrt{n})$, где $n=\operatorname{dim} \Omega$, и заметим, что интеграл $\mathscr{K}_{R}=\int_{\omega_{R}\left(y^{0}\right)}\left|w_{y}\right|^{2}\left((2 T)^{s}-|w|^{s}\right)_{+} d y$ допускает оценку

$$
\mathscr{K}_{R} \geqslant \int_{\omega_{t R}^{+}\left(y^{0}\right)}\left|w_{y}\right|^{2}(\ldots)_{+} d y+\int_{\omega_{t R}^{-}\left(y^{0}\right)}\left|w_{y}\right|^{2}(\ldots)_{+} d y=\mathscr{K}_{R}^{(1)}+\mathscr{K}_{R}^{(2)} \geqslant \mathscr{K}_{R}^{(2)} .
$$

Здесь через $(\ldots)_{+}$мы обозначили выражение $\left((2 T)^{s}-|w|^{s}\right)_{+}$,

$$
\begin{aligned}
& \omega_{t R}^{-}\left(y^{0}\right)=\left\{y \in \omega_{t R}\left(y^{0}\right):\left|w(y)-w_{t R, y^{0}}\right| \leqslant \tau\right\}, \\
& \omega_{t R}^{+}\left(y^{0}\right)=\left\{y \in \omega_{t R}\left(y^{0}\right):\left|w(y)-w_{t R, y^{0}}\right|>\tau\right\} .
\end{aligned}
$$

Заметим, что

$$
\begin{aligned}
\left|w_{t R, y^{0}}\right| & \leqslant \frac{c}{R^{\frac{n-2}{2}}}\left(\int_{\omega_{R}\left(y^{0}\right)}\left|w_{y}\right|^{2} d y\right)^{\frac{1}{2}} \stackrel{(37)}{\leqslant} c\left(\Varangle_{\omega_{R}\left(y^{0}\right)}\left|\widehat{u}_{y}\right|^{2} d y\right)^{\frac{1}{2}} \\
& \leqslant c\left\|\widehat{u}_{y}\right\|_{L^{2, n-2}\left(B_{2}^{+}\right)} \stackrel{(33)}{\leqslant} c \Theta_{2} \leqslant c_{23} .
\end{aligned}
$$


Не умаляя общности, можно считать, что $\Theta_{2} \leqslant 1$ и постоянная $c_{23}$ зависит от $\nu, \mu, a$ и $c_{\Gamma}$.

На множестве $\omega_{t R}^{-}\left(y^{0}\right)$ справедлива оценка

$$
|w(y)| \leqslant \tau+\left|w_{t R, y^{0}}\right| \leqslant \frac{T}{2^{\frac{1}{s}}}+c_{23} .
$$

Далее предположим, что $T>1$ и $s<1$ фиксированы так, чтобы выполнялось неравенство

$$
\frac{T}{2^{\frac{1}{s}}} \geqslant c_{23} \text {. }
$$

Тогда $|w(y)| \leqslant 2 T / 2^{1 / s}$ и $(2 T)^{s}-|w|^{s} \geqslant(1 / 2) T^{s}$ на $\omega_{t R}^{-}\left(y^{0}\right)$. Отсюда следует, что

$$
\mathscr{K}_{R}^{(2)} \geqslant \frac{T^{s}}{2} \int_{\omega_{t R}^{-}\left(y^{0}\right)}\left|w_{y}\right|^{2} d y .
$$

Учитывая оценку (43), после сокращения неравенства (40) на $T^{s}$ получаем неравенство

$$
\begin{aligned}
\int_{\omega_{t R}^{-}\left(y^{0}\right)}\left|w_{y}\right|^{2} d y \leqslant c_{24} & \left(s \int_{\omega_{R}\left(y^{0}\right)}\left|w_{y}\right|^{2} d y+\int_{\omega_{R}\left(y^{0}\right)}|w| \chi_{+}\left(1+\left|\widehat{u}_{y}\right|^{2}\right) d y\right. \\
& \left.+\int_{\omega_{R}\left(y^{0}\right)} \widehat{\omega}^{2}\left(R^{2},\left|\widehat{u}-\widehat{u}^{0}\right|^{2}\right)\left|\widehat{u}_{y}\right|^{2} d y\right) .
\end{aligned}
$$

Здесь $\widehat{\omega}(\cdot, \cdot)$ - ограниченная непрерывная функция, обладаюшая свойствами функции $\omega$ из условия б) теоремы 1.

Интеграл $I_{R}=\int_{\omega_{R}\left(y^{0}\right)}|w| \chi_{+}\left(1+\left|\widehat{u}_{y}\right|^{2}\right) d y$ оценим следующим образом:

$$
\begin{aligned}
& I_{R} \leqslant\left(\int_{\omega_{R}\left(y^{0}\right)}\left(1+\left|\widehat{u}_{y}\right|\right)^{p} d y\right)^{\frac{2}{p}}\left(\int_{\omega_{R}\left(y^{0}\right)}\left(|w| \chi_{+}\right)^{\frac{p}{p-2}} d y\right)^{\frac{p-2}{p}}\left|\omega_{R}\right| \\
& \stackrel{(34)}{\leqslant} c T^{\frac{4-p}{p}} \int_{\omega_{\lambda R}\left(y^{0}\right)}\left(1+\left|\widehat{u}_{y}\right|\right)^{2} d y\left(\oint_{\omega_{R}\left(y^{0}\right)}|w|^{2} d y\right)^{\frac{p-2}{p}} \\
& \stackrel{(37)}{\leqslant} c T^{\frac{4-p}{p}} \int_{\omega_{\lambda R}\left(y^{0}\right)}\left(1+\left|\widehat{u}_{y}\right|\right)^{2} d y\left(\oint_{\omega_{R}\left(y^{0}\right)}\left|\widehat{u}_{y}\right|^{2} d y\right)^{\frac{p-2}{p}} \\
& \stackrel{(33)}{\leqslant} c T^{\frac{4-p}{p}} \Theta_{2}^{\frac{2(p-2)}{p}} \int_{\omega_{\lambda R}\left(y^{0}\right)}\left(1+\left|\widehat{u}_{y}\right|\right)^{2} d y .
\end{aligned}
$$

В первом неравенстве число $p \leqslant s$ фиксировано согласно неравенству $(39), s>2-$ из оценки (34).

Оценим теперь интеграл $L_{R}=\int_{\omega_{R}\left(y^{0}\right)} \widehat{\omega}^{2}\left(R^{2},\left|\widehat{u}-\widehat{u}^{0}\right|^{2}\right)\left|\widehat{u}_{y}\right|^{2} d y$ из неравенства (44):

$$
\begin{aligned}
& L_{R} \leqslant\left(\int_{\omega_{R}\left(y^{0}\right)}\left|\widehat{u}_{y}\right|^{p} d y\right)^{\frac{2}{p}}\left|\omega_{R}\right|\left(\oint_{\omega_{R}\left(y^{0}\right)} \widehat{\omega} \frac{2 p}{p-2}\left(R^{2},\left|\widehat{u}-\widehat{u}^{0}\right|^{2}\right) d y\right)^{\frac{p-2}{p}} \\
& \stackrel{(34)}{\leqslant} c \int_{\omega_{\lambda R}\left(y^{0}\right)}\left(1+\left|\widehat{u}_{y}\right|\right)^{2} d y \widehat{\omega}^{\frac{p-2}{p}}\left(R^{2}, \oint_{\omega_{R}\left(y^{0}\right)}\left|\widehat{u}-\widehat{u}^{0}\right|^{2} d y\right)
\end{aligned}
$$




$$
\begin{aligned}
& \leqslant c \widehat{\omega}^{\frac{p-2}{p}}\left(R^{2}, c \Varangle_{\omega_{R}\left(y^{0}\right)}\left|\widehat{u}_{y}\right|^{2} d y\right) \int_{\omega_{\lambda R}\left(y^{0}\right)}\left(1+\left|\widehat{u}_{y}\right|\right)^{2} d y \\
& \stackrel{(33))}{\leqslant} c \widehat{\omega}^{\frac{p-2}{p}}\left(R^{2}, c \Theta_{2}^{2}\right) \int_{\omega_{\lambda R}\left(y^{0}\right)}\left(1+\left|\widehat{u}_{y}\right|\right)^{2} d y .
\end{aligned}
$$

Второе неравенство в этой цепочке неравенств справедливо в силу выпуклости функции $\widehat{\omega}(\cdot, \cdot)$ по второму аргументу.

Теперь из неравенства (44) следует оценка

$$
\begin{aligned}
\int_{\omega_{t R}\left(y^{0}\right)}\left|w_{y}\right|^{2} d y \leqslant & c_{25}\left(s+T^{\frac{4-p}{p}} \Theta_{2}^{\frac{2(p-2)}{p}}+\widehat{\omega}^{\frac{p-2}{p}}\left(R^{2}, c_{26} \Theta_{2}^{2}\right)\right) \\
& \times \int_{\omega_{\lambda R}\left(y^{0}\right)}\left(1+\left|\widehat{u}_{y}\right|\right)^{2} d y+\int_{\omega_{t R}^{+}\left(y^{0}\right)}\left|w_{y}\right|^{2} d y
\end{aligned}
$$

где $c_{25}$ зависит от $\nu, \mu, a, c_{\Gamma}$ и $n$, а $c_{26}=c_{26}\left(c_{\Gamma}, n\right)$.

Чтобы оценить интеграл $M_{R}=\int_{\omega_{t R}^{+}\left(y^{0}\right)}\left|w_{y}\right|^{2} d y$ из неравенства (45), нам потребуется оценка

$$
\left\|w_{y}\right\|_{L^{2, n-2}\left(\omega_{\frac{3}{4} R}\left(y^{0}\right)\right)} \leqslant c_{27} \Theta_{2} .
$$

Заметим, что для любой точки $y \in \omega_{\frac{3}{4}} R\left(y^{0}\right)$ решение $v$ задачи (35) удовлетворяет неравенствам

$$
\begin{gathered}
\int_{\omega_{\rho}(y)}\left|v_{y}\right|^{2} d y \leqslant c\left(\frac{\rho}{R}\right)^{n} \int_{\omega_{\frac{R}{4}}(y)}\left|v_{y}\right|^{2} d y \leqslant c\left(\frac{\rho}{R}\right)^{n} \int_{\omega_{R}\left(y^{0}\right)}\left|v_{y}\right|^{2} d y \\
\stackrel{(37)}{ } \leqslant\left(\frac{\rho}{R}\right)^{n} \int_{\omega_{R}\left(y^{0}\right)}\left|\widehat{u}_{y}\right|^{2} d y \quad \forall \rho \leqslant \frac{R}{4} .
\end{gathered}
$$

Первое неравенство представляет собой оценку Кампанато для решения линейной задачи с постоянными коэффициентами при нулевом условии Дирихле.

Из полученного соотношения следует, что

$$
\oint_{\omega_{\rho}(y)}\left|v_{y}\right|^{2} d y \leqslant c \Varangle_{\omega_{R}\left(y^{0}\right)}\left|\widehat{u}_{y}\right|^{2} d y \leqslant c\left\|\widehat{u}_{y}\right\|_{L^{2, n-2}\left(B_{2}^{+}\right)}^{2} \stackrel{(33)}{\leqslant} c \Theta_{2}^{2},
$$

T.e.

$$
\left\|v_{y}\right\|_{L^{2, n-2}\left(\omega_{\frac{3}{4} R}\left(y^{0}\right)\right)} \leqslant c_{28} \Theta_{2} .
$$

Неравенства (33) и (47) гарантируют справедливость оценки (46).

Продолжим функцию $w$ нулем в $B_{R}\left(y^{0}\right) \backslash \omega_{R}\left(y^{0}\right)$ и обозначим продолженную функцию $\widetilde{w}\left(\left.w\right|_{\Gamma_{R}\left(y^{0}\right)}=0\right)$. Покажем, что

$$
\omega_{t R}^{+} \subset\left\{y \in Q_{t R}\left(y^{0}\right):\left|\widetilde{w}(y)-\widetilde{w}_{t R, y^{0}}\right|>\frac{\tau}{2}\right\} \equiv Q_{t R}^{(\tau)},
$$

где $\widetilde{w}_{t R, y^{0}}=f_{Q_{t R}\left(y^{0}\right)} \widetilde{w} d y, \tau=T / 2^{1 / s}$. 
Действительно, пусть $y \in \omega_{t R}\left(y^{0}\right)$ и $\left|w(y)-w_{t R, y^{0}}\right|>\tau$. Тогда

$$
\begin{aligned}
\left|\widetilde{w}(y)-\widetilde{w}_{t R, y^{0}}\right| & =\left|\left(w(y)-w_{t R, y^{0}}\right)+\left(w_{t R, y^{0}}-\widetilde{w}_{t R, y^{0}}\right)\right| \\
& \geqslant|| w(y)-w_{t R, y^{0}}|-| w_{t R, y^{0}}-\widetilde{w}_{t R, y^{0}}||=l .
\end{aligned}
$$

Нетрудно видеть, что $\left|\widetilde{w}_{t R, y^{0}}\right| \leqslant c_{29} \Theta_{2} \leqslant c_{29}$.

Усилим условие (42) и потребуем, чтобы

$$
4 \max \left\{c_{23}, c_{29}\right\} \leqslant \tau \text {. }
$$

Тогда $\left|w_{t R, y^{0}}-\widetilde{w}_{t R, y^{0}}\right| \leqslant \tau / 2$, и неравенство $\left(48^{\prime}\right)$ можно продолжить:

$$
l \geqslant \tau-\frac{\tau}{2}=\frac{\tau}{2}
$$

т. е. вложение (48) верно.

Интеграл $M_{R}$ оценим следующим образом:

$$
\begin{aligned}
& M_{R} \leqslant\left(\oint_{\omega_{t R}^{+}\left(y^{0}\right)}\left|w_{y}\right|^{p} d y\right)^{\frac{2}{p}}\left|\omega_{t R}^{+}\right| \stackrel{(34),(39)}{\leqslant} c \int_{\omega_{\lambda R}\left(y^{0}\right)}\left(1+\left|\widehat{u}_{y}\right|\right)^{2} d y\left(\frac{\left|\omega_{t R}^{+}\right|}{\left|\omega_{\lambda R}\right|}\right)^{1-\frac{2}{p}} \\
& \quad \stackrel{(48)}{\leqslant} c\left(\frac{\left|Q_{t R}^{(\tau)}\right|}{\left|\omega_{\lambda R}\right|}\right)^{1-\frac{2}{p}} \int_{\omega_{\lambda R}\left(y^{0}\right)}\left(1+\left|\widehat{u}_{y}\right|\right)^{2} d y .
\end{aligned}
$$

Как было показано $\Phi$. Йоном и Л. Ниренбергом в работе [9], функция $\widetilde{w}$ из $\mathscr{L}^{2, n}\left(Q_{t R}\left(y^{0}\right)\right)$ обладает следуюшим свойством: сушествуют постоянные $H_{0}$ и $\beta>0$, зависящие только от размерности $n$, такие, что

$$
\frac{\left|Q_{t R}^{(\tau)}\right|}{\left|Q_{t R}\right|} \leqslant H \exp \left(-\frac{\beta \tau}{2[\widetilde{w}]_{2, n ; Q_{t R}}}\right)
$$

Заметим, что $Q_{t R}\left(y^{0}\right) \subset B_{\frac{3}{4} R}\left(y^{0}\right)$ и

$$
[\widetilde{w}]_{2, n ; Q_{t R}} \leqslant c\left\|\widetilde{w}_{y}\right\|_{L^{2, n-2}\left(Q_{t R}\right)} \leqslant c\left\|w_{y}\right\|_{L^{2, n-2}\left(\omega_{\frac{3}{4} R}\left(y^{0}\right)\right)} \stackrel{(46)}{\leqslant} c_{30} \Theta_{2} .
$$

Теперь из неравенства (50) следует, что

$$
M_{R} \leqslant c_{31} \exp \left(-\frac{c_{32} \tau}{\Theta_{2}}\right) \int_{\omega_{\lambda R}\left(y^{0}\right)}\left(1+\left|\widehat{u}_{y}\right|\right)^{2} d y
$$

Учитывая оценку (51), из (45) выводим неравенство

$$
\begin{aligned}
\int_{\omega_{t R}}\left|w_{y}\right|^{2} d y \leqslant & c_{33}\left[s+T^{\frac{4-p}{p}} \Theta_{2}^{\frac{2(p-2)}{p}}+\widehat{\omega}^{\frac{p-2}{p}}\left(R^{2}, c_{26} \Theta_{2}^{2}\right)+\exp \left(-\frac{c_{32} \tau}{\Theta_{2}}\right)\right] \\
& \times \int_{\omega_{\lambda R}\left(y^{0}\right)}\left(1+\left|\widehat{u}_{y}\right|\right)^{2} d y .
\end{aligned}
$$


Для функции $\psi\left(\rho, y^{0}\right)=\int_{\omega_{\rho}\left(y^{0}\right)}\left(1+\left|\widehat{u}_{y}\right|\right)^{2} d y$ из (36) и $(52)$ следует оценка

$$
\begin{aligned}
\psi\left(\rho, y^{0}\right) \leqslant & c_{34}\left\{\left(\frac{\rho}{R}\right)^{n}+\left[s+T^{\frac{4-p}{p}} \Theta_{2}^{\frac{2(p-2)}{p}}\right.\right. \\
& \left.\left.+\widehat{\omega}^{\frac{p-2}{p}}\left(R^{2}, c_{26} \Theta_{2}^{2}\right)+\exp \left(-\frac{c_{32} \tau}{\Theta_{2}}\right)\right]\right\} \psi\left(\lambda R, y^{0}\right) \quad \forall \rho \leqslant R .
\end{aligned}
$$

Очевидно, что неравенства вида (53) справедливы и при $\rho \in(R, \lambda R)$. Далее мы заменим обозначение $\lambda R$ на $R$, считая $R \leqslant 1 / 2$. Таким образом, для всех $\rho \leqslant R$ верны неравенства

$$
\psi\left(\rho, y^{0}\right) \leqslant c_{35}\left\{\left(\frac{\rho}{R}\right)^{n}+[\ldots]\right\} \psi\left(\rho, y^{0}\right)
$$

где через $[\ldots]$ обозначено выражение, стоящее в квадратных скобках неравенства (53).

Согласно известной лемме Кампанато (см., например, [10, гл. II, лемма 2.1]) для любого $\varepsilon>0$ сушествует число $\delta_{0}=\delta_{0}\left(c_{35}, n, \varepsilon\right)$ такое, что если в неравенстве $(54)$

$$
[\ldots]<\delta_{0}
$$

TO

$$
\psi\left(\rho, y^{0}\right) \leqslant c_{36}\left(\frac{\rho}{R}\right)^{n-\varepsilon} \psi\left(R, y^{0}\right)
$$

с постоянной $c_{36}$, зависящей от $c_{35}, n$ и $\varepsilon$.

Пусть $\varepsilon>0$ фиксировано произвольно. Зафиксируем теперь $s<1$ из условия

$$
s<\frac{\delta_{0}}{4} .
$$

Поскольку $\Theta_{2} \leqslant 1$, то

$$
\exp \left(-\frac{c_{32} \tau}{\Theta_{2}}\right) \leqslant \exp \left(-c_{32} \tau\right)=\exp \left(-\frac{c_{32} T}{2^{1 / s}}\right)
$$

Выберем теперь $T>1$ так, чтобы кроме неравенства (49) выполнялось условие

$$
\exp \left(\frac{c_{32} T}{2^{1 / s}}\right)<\frac{\delta_{0}}{4}
$$

Осталось зафиксировать числа $\Theta_{2} \leqslant \Theta_{1}$ и $R_{1} \leqslant 1 / 2$ так, чтобы для $R \leqslant R_{1}$ было верно неравенство

$$
T^{\frac{4-p}{p}} \Theta_{2}^{\frac{2(p-2)}{p}}+\widehat{\omega}^{\frac{p-2}{p}}\left(R^{2}, c_{26} \Theta_{2}^{2}\right)<\frac{\delta_{0}}{2} .
$$

Условия (57)-(59) гарантируют справедливость неравенства (55), а следовательно, и оценки (56). 
Поскольку точка $y^{0} \in \overline{B_{1}^{+}(0)}$ была фиксирована произвольно, то из оценки $(56)$ при $R=R_{1} \leqslant 1 / 2$ следует, что

$$
\left\|\widehat{u}_{y}\right\|_{L^{2, n-\varepsilon}\left(B_{1}^{+}\right)} \leqslant c_{37}\left(1+\left\|\widehat{u}_{y}\right\|_{2, B_{2}^{+}}\right)
$$

где постоянная $c_{37}$ зависит от $\nu, \mu, a, n, c_{\Gamma}, \varepsilon$, модуля непрерьвности функций $\mathfrak{O}_{k l}^{\gamma \delta}$ и $R_{1}^{-1}$.

Из (60) следует, что

$$
\|\widehat{u}\|_{\mathscr{L}^{2, n+2-\varepsilon}\left(B_{1}^{+}\right)} \leqslant c_{38}\left(1+\|\widehat{u}\|_{W_{2}^{1}\left(B_{2}^{+}\right)}\right),
$$

и, согласно изоморфизму пространств $\mathscr{L}^{2, n+2-\varepsilon}(\cdot)$ и $C^{\alpha}(\cdot)$ при $\alpha=1-\varepsilon / 2$, получаем оценку

$$
\|\widehat{u}\|_{C^{\alpha}\left(\overline{B_{1}^{+}}\right)} \leqslant c_{39}\left(1+\|\widehat{u}\|_{W_{2}^{1}\left(B_{2}^{+}\right)}\right) .
$$

Постоянные $c_{38}$ и $c_{39}$ зависят от тех же величин, что и $c_{37}$.

Из оценки (62) следует справедливость утверждения леммы 2 для функции $u(x)=\widehat{u}(y(x))$.

Согласно замечанию 3 из лемм 1 и 2 следует справедливость утверждения теоремы 1 .

\section{Список литературы}

1. Frehse J. On two-dimensional quasi-linear elliptic systems // Manuscripta Math. 1979. V. 28. P. 21-50.

2. Apxuпова A. A. Проблема разрешимости для недиагональных эллиптических систем с квадратичной нелинейностью по градиенту (двумерных случай) // Зап. научн. семин. ПОМИ. 2003. Т. 295. С. 4-17.

3. Giaquinta M., Giusti E. Non linear elliptic systems with quadratic growth // Manuscripta Math. 1978. V. 24. P. 323-349.

4. Hamburger $C$. A new partial regularity proof for solutions of nonlinear elliptic systems // Manuscripta Math. 1998. V. 95. № 1. P. 11-31.

5. Архипова A. A. О регулярности решений краевых задач для квазилинейных эллиптических систем с квадратичной нелинейностью // Сб. "Проблемы математического анализа". Вып. 15. С.-Пб.: Изд-во С.-Петербургского университета, 1995. С. 47-69.

6. Arkhipova A. A. Quasireverse Holder inequalities and a priori estimates for quasilinear elliptic systems // Rendic. Mat. Acc. Lincei. 2003. V. 14. P. 9.

7. Kufner A., John O., Fučik S. Functional Spaces. Prague: Academia Prague, 1977.

8. Troiniello G. M. Elliptic Differential Equations and Obstacle Problems. N. Y.: Plenum Press, 1987.

9. John F., Nirenberg L. On functions of bounded mean oscillation // Comm. Pure Appl. Math. 1961. V. 17. № 3. P. 415-426.

10. Giaquinta M. Multiple Integrals in the Calculus of Variations and Nonlinear Elliptic Systems. Princeton: Princeton Univ. Press, 1983.

Санкт-Петербургский государственный университет

Поступило в редакцию 25.09 .2003 